\title{
RESPONSE OF CARAWAY YIELD TO PLANT DISTANCE AND NITROGEN FERTILIZATION TREATMENTS
}

\author{
(Received: 9 .11 .2009)
}

\author{
By \\ A.A. El-Sayed, S.M. Nasr*, M.A. Darwesh and M.M. Soliman* \\ Ornamental Horticulture Department, Fac. of Agric., Cairo Univ., and * Laboratory for Design and \\ Statistical Analysis, Agricultural Research Center, Giza, Egypt.
}

\begin{abstract}
Two field experiments were carried out at the Agricultural Experiment and Research Station, Faculty of Agriculture, Cairo University, during the 2006-2007 and 2007-2008 seasons to study the effect of plant distance and nitrogen fertilization on the yield of caraway (Carum carvi L.). Plant distance treatments were 20, 40 and $60 \mathrm{~cm}$. Nitrogen fertilization treatments were $0,10,20,30,40$ and $45 \mathrm{~kg} \mathrm{~N} / \mathrm{feddan}$. The results indicated that the maximum values for plant height were obtained when the distance was $20 \mathrm{~cm}$ between the plants, while for the number of branches/plant, the number of umbels/plant, fruit weight/plant $(\mathrm{gm})$, fruit yield/plot $(\mathrm{gm})$, fruit yield/feddan $(\mathrm{kg}), 1000$ seeds weight $(\mathrm{gm})$, oil\% and oil yield/feddan (L) the maximum values were obtained when the distance was $60 \mathrm{~cm}$ between the plants. Also, the results indicated that the maximum values for plant height, number of branches/plant, number of umbels/plant, fruit weight/plant (gm), fruit yield/plot (gm), fruit yield/feddan $(\mathrm{kg})$, weight of 1000 seeds $(\mathrm{gm})$, oil\% and oil yield/feddan (L) were obtained with applying $40 \mathrm{~kg}$ of nitrogen/feddan. Interaction between plant distance and nitrogen fertilization resulted in the maximum values of fruit yield/feddan with the distance of $40 \mathrm{~cm}$ between plants and applying $40 \mathrm{~kg}$ nitrogen/feddan.
\end{abstract}

Key words: caraway, Carum carvi, distance, nitrogen fertilizer.

\section{INTRODUCTION}

Medicinal and aromatic plants are very important economic plants. Nowadays, there is a return to the use of natural products in pharmaceuticals and cosmetics. Caraway (Carum carvi L.), a member of the Umbelliferae (Apiaceae), is an annual spice crop. It has an erect, branched stem. The plant forms a shallow tap root with minimal branching. White flowers are formed at the terminal buds. The oil content and composition are influenced by crop maturity, cultivar and growing conditions. Fertilizers are chemical compounds applied to promote plant growth and fruit production. This application is either through the soil (for uptake by plant roots) or sprayed on the foliage of the plant. The distance of planting affects the vegetative growth. Radwan (1980) mentioned that there was a significant decrease in caraway plant height with increasing plant spacing. Badran and Hafez (2002) indicated that reducing Nigella sativa plant density caused considerable increase in each of branch number, herb dry weight as well as fruit yield/plant, while plant height and fruit yield/feddan were greatly reduced. Planting distance affects the essential oil yield. Radwan (1980) obtained an increase in caraway oil yield/plot as a result of growing the plants at a distance of $20 \mathrm{~cm}$. Ahmed (1997) found that the volatile and fixed oil percentages in the seeds of Nigella sativa were increased with increasing the distance between the plants up to 40 $\mathrm{cm}$. The vegetative growth and herb yield are affected with nitrogen fertilizers. Radwan (1980) found that the plant height, number of branches, umbels/plant and fresh and dry weights of coriander and caraway were increased as the levels of nitrogen increased. As regards to the effect of nitrogenous fertilizers on oil yield, Rahman et al. (1990), Barreyro et al. (1993) and Hussien (1995) found that increasing nitrogenous fertilization for Coriandrum sativum increased essential oil yield. Bhati and Shaktawat (1994) and Tiwari and Banafar (1995) stated that the application of $60 \mathrm{~kg}$ nitrogen/ha increased the essential oil yield of coriander plant.

The objective of the present study was to find out the effect of plant distance and nitrogen fertilization treatments on the yield of caraway.

\section{MATERIALS AND METHODS}

Two field experiments were carried out at the Agricultural Experiment and Research Station, 
Faculty of Agriculture, Cairo University, during the 2006-2007 and 2007-2008 seasons to study the effect of plant distance and nitrogen fertilization on the yield of caraway (Carum carvi L.). The experimental design used was a split-plot with three replications. Plant distance treatments were 20,40 and $60 \mathrm{~cm}$, assigned to the main plots. Nitrogen fertilization treatments were $0,10,20$, 30,40 and $45 \mathrm{~kg} \mathrm{~N} /$ feddan, assigned to the subplots. The field was prepared for cultivation, by deep ploughing and dividing into 54 plots $(2 \times 2$ $\mathrm{m})$, each plot contained 3 rows of $60 \mathrm{~cm}$ width. Caraway seeds were sown on one side of each row on $21^{\text {st }}$ September and $1^{\text {st }}$ October 2006 and 2007 in the two seasons, respectively. Nitrogen fertilization as ammonium sulfate $\left(\left(\mathrm{NH}_{4}\right)_{2} \mathrm{SO}_{4}\right)$ was divided into two equal portions. The first portion was added after 30 days from sowing. The other one was added after a month from the first. In the second season, the oil percentage was determined in fruits, which were crushed half an hour before distillation, according to the procedure described by the British Pharmacopoeia (1963).

In both season, data were recorded for the following growth characters:

- Plant height $(\mathrm{cm})$

- Number of branches per plant

- Number of umbels per plant

- Number of umbelet per umbel

- Number of fruits per umbelet

- Weight of fruits (gm/plant)

- Fruit yield per plot (gm/plot)

- Fruit yield per feddan (kg/feddan)

- Fruit index (weight of 1000 fruit)

- Essential oil percentage in the fruits

- Essential oil yield / plant (ml)

- Essential oil yield /feddan (L)

All data were subjected to statistical analysis according to the procedures (ANOVA) reported by Snedecor and Cochran (1980). Combined analysis over the two seasons of experimentation was done after testing the homogeneity of variance between the two seasons. Treatment means were compared by the LSD Test at the 5\% level of probability.

\section{RESULTS AND DISCUSSION}

\subsection{Plant distance effect}

Data presented in Tables (1,6 and 7) reveal that plant height, number of branches per plant, number of umbels per plant, fruit yield per plot (gm), fruit yield per feddan $(\mathrm{kg})$, oil percentage and oil yield per feddan (L) traits were significantly affected by changing the distance between plants. In the plant height trait, the maximum value was obtained by using $20 \mathrm{~cm}$ between plants, while in the other traits the maximum values were obtained by using $60 \mathrm{~cm}$. Plants grown at distances of 40 and $60 \mathrm{~cm}$ showed significant difference in the number of umbels/plant, fruit yield/plot (gm), fruit yield/feddan $(\mathrm{kg})$, oil \% and oil yield/feddan (L). These results are in agreement with those obtained by Radwan (1980) on umbelliferous plants and Ahmed (1997) on Nigella sativa.

\subsection{Nitrogen fertilizer effect}

Data presented in Tables (1, 6 and 7) reveal that plant height, number of branches per plant, number of umbels per plant, weight of fruits per plant (gm), fruit yield per plot (gm), fruit yield per feddan $(\mathrm{kg})$, fruit index (weight of 1000 fruits (gm)), oil percentage and oil yield per feddan (L) were significantly affected by changing the nitrogen fertilization. In the case of plant height and number of umbels per plant, the maximum values were obtained by applying $40 \mathrm{~kg} \mathrm{~N} /$ feddan, while in case of number of branches per plant, weight of fruits per plant (gm), fruit yield per plot $(\mathrm{gm})$, fruit yield per feddan $(\mathrm{kg})$, fruit index (weight of 1000 fruits (gm)) and oil yield per feddan (L) it was found that the maximum values were obtained by applying $40 \mathrm{~kg} \mathrm{~N} /$ feddan. There were no significant differences between applying 30 and $40 \mathrm{~kg} \mathrm{~N} /$ feddan in the number of branches/plant, fruit yield/feddan $(\mathrm{kg})$ and weight of 1000 fruits $(\mathrm{gm})$. Concerning the weight of seeds/plant $(\mathrm{gm})$, there were no significant differences between applying 30, 40 and $45 \mathrm{~kg}$ $\mathrm{N} /$ feddan. The maximum value of oil percentage was obtained from applying $40 \mathrm{~kg}$ nitrogen/feddan and there were no significant differences between the applications 20,30 and $40 \mathrm{~kg}$ nitrogen per feddan. These results are in agreement with those obtained by El-Mansi et al. (1970) on coriander and Radwan (1980) on umbelliferous plants.

\subsection{Seasonal variation}

Data presented in Table (1) reveal that plant height, number of branches per plant, number of umbels per plant, weight of fruits per plant (gm), fruit yield per plot $(\mathrm{gm})$ and fruit yield per feddan (kg) traits were significantly affected by the season. These results may be due to the environmental conditions.

\subsection{The interaction between season and plant distance}

Data presented in Table (4) reveal that weight of fruits per plant (gm), fruit yield per plot (gm) and fruit yield per feddan $(\mathrm{kg})$ traits were significantly affected by the interaction between seasons and plant distance. No significant differences in weight of fruits/plant (gm) were obtained when using 40 and $60 \mathrm{~cm}$ between plants. 
Table (1): Effect of plant distance, nitrogen fertilization and seasonal variation on growth and productivity of caraway (Carum carvi L.)

\begin{tabular}{|c|c|c|c|c|c|c|c|c|c|c|}
\hline \multicolumn{2}{|c|}{ Studied Factors } & \multirow{2}{*}{$\begin{array}{c}\begin{array}{c}\text { Plant } \\
\text { height } \\
(\mathrm{cm})\end{array} \\
113.30\end{array}$} & \multirow{2}{*}{$\begin{array}{c}\begin{array}{c}\text { Number of } \\
\text { branches / } \\
\text { plant }\end{array} \\
12.09\end{array}$} & \multirow{2}{*}{$\begin{array}{c}\text { Number of } \\
\text { umbels / } \\
\text { plant }\end{array}$} & \multirow{2}{*}{$\begin{array}{c}\begin{array}{c}\text { Number of } \\
\text { umbelet } / \\
\text { umbel }\end{array} \\
15.48\end{array}$} & \multirow{2}{*}{$\begin{array}{c}\text { Number of } \\
\text { fruits / } \\
\text { umbelet } \\
\mathbf{8 . 0 2}\end{array}$} & \multirow{2}{*}{$\begin{array}{c}\begin{array}{c}\text { Weight of } \\
\text { fruits/plant } \\
\text { (gm) }\end{array} \\
25.23\end{array}$} & \multirow{2}{*}{$\begin{array}{l}\text { Yield /plot } \\
\begin{array}{l}\text { (gm) } \\
\end{array} \\
301.48\end{array}$} & \multirow{2}{*}{$\begin{array}{c}\begin{array}{c}\text { Yield } \\
\text { /feddan } \\
(\mathrm{kg})\end{array} \\
316.55\end{array}$} & \multirow{2}{*}{$\begin{array}{c}\begin{array}{c}\text { Weight of } \\
1000 \\
\text { fruits (gm) }\end{array} \\
4.44\end{array}$} \\
\hline Seasons & $2006 / 2007$ & & & & & & & & & \\
\hline & $2007 / 2008$ & 94.10 & 9.37 & 43.85 & 14.16 & 7.61 & 8.12 & 163.04 & 171.19 & 4.00 \\
\hline \multicolumn{2}{|c|}{ F test } & $*$ & $*$ & $*$ & - & - & $*$ & $*$ & $*$ & - \\
\hline \multicolumn{2}{|c|}{ LSD at 0.05} & 8.17 & 0.84 & 14.23 & $\mathbf{N S}$ & NS & 16.83 & 50.18 & 71.25 & $\mathbf{N S}$ \\
\hline & $20 \mathrm{~cm}$ & 110.44 & 9.50 & 38.66 & 14.46 & 8.03 & 12.18 & 185.836 & 195.12 & 3.71 \\
\hline Plant & $40 \mathrm{~cm}$ & 104.22 & 10.75 & 59.40 & 15.22 & 7.98 & 18.01 & 236.417 & 248.23 & 4.30 \\
\hline Distance & $60 \mathrm{~cm}$ & 96.58 & 11.90 & 70.69 & 14.78 & 7.43 & 19.83 & 274.530 & 288.25 & 4.66 \\
\hline \multicolumn{2}{|c|}{ F test } & $*$ & $*$ & $*$ & - & - & - & $*$ & $*$ & - \\
\hline \multicolumn{2}{|c|}{ LSD at 0.05} & 9.97 & 1.113 & 15.07 & NS & NS & NS & 69.360 & 47.98 & NS \\
\hline \multirow{6}{*}{$\begin{array}{c}\text { Nitrogen } \\
\text { Fertilization }\end{array}$} & 0 kg N/feddan & 93.60 & 8.33 & 38.22 & 14.88 & 7.46 & 9.74 & 150.00 & 157.50 & 3.789 \\
\hline & $10 \mathrm{~kg} \mathrm{~N} /$ feddan & 101.30 & 9.66 & 48.96 & 14.51 & 7.43 & 14.60 & 185.34 & 194.61 & 4.158 \\
\hline & $20 \mathrm{~kg} \mathrm{~N} /$ feddan & 105.30 & 10.88 & 57.88 & 14.82 & 7.90 & 16.84 & 237.56 & 249.44 & 4.330 \\
\hline & $30 \mathrm{~kg}$ N/feddan & 108.70 & 12.16 & 62.08 & 14.89 & 7.53 & 18.53 & 277.72 & 291.60 & 4.538 \\
\hline & $40 \mathrm{~kg} \mathrm{~N} /$ feddan & 113.10 & 13.38 & 72.41 & 14.77 & 7.90 & 22.45 & 337.66 & 354.54 & 4.629 \\
\hline & $45 \mathrm{~kg}$ N/feddan & 100.20 & 9.944 & 57.95 & 15.04 & 8.66 & 17.88 & 205.25 & 215.52 & 3.917 \\
\hline \multicolumn{2}{|c|}{ F test } & $*$ & $*$ & $*$ & - & - & $*$ & $*$ & * & $*$ \\
\hline \multicolumn{2}{|c|}{ LSD at 0.05} & 3.34 & 1.24 & 8.91 & NS & NS & 6.93 & 61.83 & 69.43 & 0.180 \\
\hline
\end{tabular}


Table (2): Effect of the interaction between plant distance, nitrogen fertilization and seasonal variation on growth and productivity of caraway (Carum carvi L.)

\begin{tabular}{|c|c|c|c|c|c|c|c|c|c|c|c|c|c|c|c|c|c|c|c|}
\hline \multirow[b]{2}{*}{$\begin{array}{c}\text { Plant } \\
\text { distance }\end{array}$} & \multirow[b]{2}{*}{$\begin{array}{c}\text { Nitrogen } \\
\text { Fertilization }\end{array}$} & \multicolumn{2}{|c|}{$\begin{array}{c}\text { Plant height } \\
\text { (cm) }\end{array}$} & \multicolumn{2}{|c|}{$\begin{array}{c}\text { Number of } \\
\text { branches } \\
\text { /plant }\end{array}$} & \multicolumn{2}{|c|}{$\begin{array}{c}\text { Number of } \\
\text { umbels /plant }\end{array}$} & \multicolumn{2}{|c|}{$\begin{array}{c}\text { Number of } \\
\text { umbelet /umbel }\end{array}$} & \multicolumn{2}{|c|}{$\begin{array}{l}\text { Number of } \\
\text { fruits/ } \\
\text { umbelet }\end{array}$} & \multicolumn{2}{|c|}{$\begin{array}{c}\text { Weight of } \\
\text { fruits /plant } \\
\text { (gm) }\end{array}$} & \multicolumn{2}{|c|}{ Yield /plot (gm) } & \multicolumn{2}{|c|}{$\begin{array}{c}\text { Yield /feddan } \\
(\mathrm{kg})\end{array}$} & \multicolumn{2}{|c|}{$\begin{array}{c}\text { Weight of } \\
1000 \text { Fruits } \\
\text { (gm) }\end{array}$} \\
\hline & & $\begin{array}{l}2006 / \\
2007\end{array}$ & $\begin{array}{l}2007 / \\
2008\end{array}$ & $\begin{array}{l}2006 / \\
2007\end{array}$ & $\begin{array}{l}2007 / \\
2008\end{array}$ & $\begin{array}{l}2006 / \\
2007\end{array}$ & $\begin{array}{l}2007 / \\
2008\end{array}$ & $\begin{array}{l}2006 / \\
2007\end{array}$ & $\begin{array}{l}2007 / \\
2008\end{array}$ & $\begin{array}{l}2006 / \\
2007\end{array}$ & $\begin{array}{l}2007 / \\
2008\end{array}$ & $\begin{array}{l}2006 / \\
2007\end{array}$ & $\begin{array}{l}2007 / \\
2008\end{array}$ & $\begin{array}{l}2006 / \\
2007\end{array}$ & $\begin{array}{l}2007 / \\
2008\end{array}$ & $\begin{array}{l}2006 / \\
2007\end{array}$ & $\begin{array}{l}2007 / \\
2008\end{array}$ & $\begin{array}{l}2006 / \\
2007\end{array}$ & $\begin{array}{l}2007 / \\
2008\end{array}$ \\
\hline $20 \mathrm{~cm}$ & $\begin{array}{l}0 \mathrm{~kg} \text { N/feddan } \\
10 \mathrm{~kg} \text { N/feddan } \\
20 \mathrm{~kg} \text { N/feddan } \\
30 \mathrm{~kg} \text { N/feddan } \\
40 \mathrm{~kg} \mathrm{~N} / f e d d a n \\
45 \mathrm{~kg} \mathrm{~N} / \mathrm{feddan}\end{array}$ & \begin{tabular}{l|}
111.6 \\
118.6 \\
122.6 \\
128.0 \\
132.3 \\
116.6
\end{tabular} & \begin{tabular}{|c|}
92.6 \\
97.6 \\
102.6 \\
105.0 \\
107.6 \\
89.6 \\
\end{tabular} & \begin{tabular}{|c|}
9.33 \\
9.66 \\
11.00 \\
12.00 \\
13.66 \\
10.33
\end{tabular} & \begin{tabular}{|l|}
4.66 \\
7.00 \\
8.66 \\
10.00 \\
10.00 \\
7.66
\end{tabular} & \begin{tabular}{l|}
38.20 \\
42.93 \\
43.46 \\
44.33 \\
65.53 \\
59.26
\end{tabular} & \begin{tabular}{l|}
15.40 \\
21.33 \\
33.13 \\
34.20 \\
36.80 \\
29.33
\end{tabular} & $\begin{array}{l}14.66 \\
15.86 \\
15.33 \\
14.40 \\
15.00 \\
14.86\end{array}$ & \begin{tabular}{l|}
14.26 \\
13.30 \\
14.00 \\
13.86 \\
13.10 \\
14.86
\end{tabular} & $\begin{array}{l}8.20 \\
7.40 \\
9.40 \\
6.80 \\
8.60 \\
9.60\end{array}$ & $\begin{array}{l}7.60 \\
6.80 \\
7.00 \\
7.00 \\
8.40 \\
9.60\end{array}$ & $\begin{array}{l}11.90 \\
13.30 \\
16.32 \\
17.18 \\
24.39 \\
24.25\end{array}$ & \begin{tabular}{r|}
2.65 \\
4.31 \\
5.28 \\
7.44 \\
10.02 \\
9.16
\end{tabular} & $\begin{array}{l}183.33 \\
182.33 \\
276.33 \\
284.33 \\
340.66 \\
223.66\end{array}$ & \begin{tabular}{|c|}
88.33 \\
120.70 \\
133.33 \\
140.00 \\
179.66 \\
77.33
\end{tabular} & $\begin{array}{l}192.50 \\
191.45 \\
290.15 \\
298.55 \\
357.70 \\
234.85\end{array}$ & \begin{tabular}{|c|}
92.75 \\
126.73 \\
140.00 \\
147.00 \\
188.65 \\
81.20
\end{tabular} & \begin{tabular}{|l|}
3.365 \\
3.983 \\
4.055 \\
4.125 \\
4.325 \\
3.776
\end{tabular} & $\begin{array}{l}2.623 \\
3.280 \\
3.653 \\
3.813 \\
4.160 \\
3.413\end{array}$ \\
\hline $40 \mathrm{~cm}$ & $\begin{array}{l}0 \mathrm{~kg} \text { N/feddan } \\
10 \mathrm{~kg} \text { N/feddan } \\
20 \mathrm{~kg} \text { N/feddan } \\
30 \mathrm{~kg} \text { N/feddan } \\
40 \mathrm{~kg} \mathrm{~N} / f e d d a n \\
45 \mathrm{~kg} \mathrm{~N} / f e d d a n\end{array}$ & $\begin{array}{l}107.6 \\
112.0 \\
116.0 \\
120.0 \\
121.6 \\
105.3\end{array}$ & \begin{tabular}{|c|}
88.0 \\
93.6 \\
93.0 \\
96.0 \\
103.0 \\
94.3 \\
\end{tabular} & \begin{tabular}{r|}
10.33 \\
11.00 \\
11.66 \\
13.33 \\
15.00 \\
11.00
\end{tabular} & \begin{tabular}{|l|l|}
6.33 & \\
8.66 & \\
9.66 & \\
10.66 & \\
12.33 & 9.00 \\
\end{tabular} & $\begin{array}{l}52.80 \\
58.86 \\
76.46 \\
77.73 \\
92.53 \\
67.93 \\
\end{array}$ & \begin{tabular}{l|}
26.20 \\
34.66 \\
50.80 \\
53.50 \\
65.86 \\
55.53
\end{tabular} & $\begin{array}{l}15.13 \\
15.13 \\
15.40 \\
17.26 \\
15.33 \\
14.93\end{array}$ & \begin{tabular}{|l|}
16.20 \\
14.66 \\
14.00 \\
15.10 \\
14.86 \\
14.66
\end{tabular} & $\begin{array}{l}7.60 \\
8.00 \\
7.20 \\
7.60 \\
8.80 \\
8.00\end{array}$ & $\begin{array}{l}8.40 \\
8.40 \\
7.60 \\
7.00 \\
7.60 \\
9.60\end{array}$ & $\begin{array}{l}17.11 \\
25.79 \\
29.41 \\
30.83 \\
36.93 \\
26.50\end{array}$ & $\begin{array}{l}3.35 \\
6.22 \\
8.53 \\
10.2 \\
11.3 \\
9.81\end{array}$ & $\begin{array}{l}188.33 \\
222.00 \\
278.00 \\
378.66 \\
471.33 \\
266.00\end{array}$ & \begin{tabular}{l|}
92.00 \\
121.60 \\
180.07 \\
226.66 \\
258.66 \\
153.66
\end{tabular} & $\begin{array}{l}197.75 \\
233.10 \\
291.90 \\
397.60 \\
494.90 \\
279.30\end{array}$ & $\begin{array}{l}96.60 \\
127.68 \\
189.08 \\
238.00 \\
271.59 \\
161.35\end{array}$ & $\begin{array}{l}3.995 \\
4.603 \\
4.751 \\
5.041 \\
4.724 \\
4.012\end{array}$ & $\begin{array}{l}3.980 \\
4.067 \\
4.140 \\
4.353 \\
4.450 \\
3.553\end{array}$ \\
\hline $60 \mathrm{~cm}$ & $\begin{array}{l}0 \mathrm{~kg} \text { N/feddan } \\
10 \mathrm{~kg} \text { N/feddan } \\
20 \mathrm{~kg} \text { N/feddan } \\
30 \mathrm{~kg} \text { N/feddan } \\
40 \mathrm{~kg} \mathrm{~N} / \text { feddan } \\
45 \mathrm{~kg} \mathrm{~N} / \text { feddan }\end{array}$ & \begin{tabular}{r|}
95.3 \\
100.0 \\
104.0 \\
108.3 \\
112.3 \\
107.0
\end{tabular} & \begin{tabular}{|c|}
66.6 \\
86.0 \\
93.6 \\
95.3 \\
102.0 \\
88.3 \\
\end{tabular} & $\begin{array}{l}11.33 \\
12.00 \\
13.33 \\
14.66 \\
16.00 \\
12.00\end{array}$ & $\begin{array}{l}8.00 \\
9.66 \\
11.00 \\
12.33 \\
13.33 \\
9.66\end{array}$ & $\begin{array}{l}56.46 \\
85.06 \\
87.60 \\
101.5 \\
106.3 \\
78.80\end{array}$ & $\begin{array}{r}40.30 \\
50.90 \\
55.86 \\
61.20 \\
67.40 \\
56.86\end{array}$ & $\begin{array}{l}15.73 \\
15.53 \\
15.80 \\
15.66 \\
16.73 \\
15.86\end{array}$ & $\begin{array}{l}13.33 \\
12.60 \\
14.40 \\
13.06 \\
13.60 \\
15.06\end{array}$ & $\begin{array}{l}6.80 \\
7.60 \\
9.60 \\
8.00 \\
7.20 \\
8.00\end{array}$ & $\begin{array}{l}6.20 \\
6.40 \\
6.60 \\
8.80 \\
6.80 \\
7.20\end{array}$ & $\begin{array}{l}18.98 \\
29.32 \\
32.23 \\
33.45 \\
39.13 \\
27.19\end{array}$ & $\begin{array}{c}4.45 \\
8.64 \\
9.28 \\
12.05 \\
12.89 \\
10.38 \\
\end{array}$ & $\begin{array}{l}232.66 \\
309.00 \\
364.33 \\
390.00 \\
512.33 \\
323.33\end{array}$ & $\begin{array}{l}115.36 \\
156.44 \\
193.33 \\
246.66 \\
263.33 \\
187.55\end{array}$ & $\begin{array}{l}244.30 \\
324.45 \\
382.55 \\
409.50 \\
537.95 \\
339.50\end{array}$ & $\begin{array}{l}121.12 \\
164.26 \\
203.00 \\
259.00 \\
276.50 \\
196.93\end{array}$ & $\begin{array}{l}4.639 \\
4.799 \\
4.859 \\
5.127 \\
5.466 \\
4.363\end{array}$ & $\begin{array}{l}4.133 \\
4.220 \\
4.520 \\
4.767 \\
4.650 \\
4.387\end{array}$ \\
\hline \multicolumn{2}{|c|}{ F-test } & \multicolumn{2}{|c|}{ 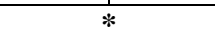 } & \multicolumn{2}{|c|}{-} & \multicolumn{2}{|c|}{ - } & \multicolumn{2}{|c|}{-} & & & & & \\
\hline \multicolumn{2}{|c|}{ LSD at 0.05} & \multicolumn{2}{|c|}{5.96} & \multicolumn{2}{|c|}{ NS } & \multicolumn{2}{|c|}{ NS } & \multicolumn{2}{|c|}{ NS } & \multicolumn{2}{|c|}{ NS } & \multicolumn{2}{|c|}{ NS } & \multicolumn{2}{|c|}{ NS } & \multicolumn{2}{|c|}{ NS } & \multicolumn{2}{|c|}{ NS } \\
\hline
\end{tabular}


Table (3): Effect of the interaction between nitrogen fertilization and seasonal variation on growth and productivity of caraway

\begin{tabular}{|c|c|c|c|c|c|c|c|c|c|c|c|c|c|c|c|c|c|c|}
\hline \multirow{2}{*}{$\begin{array}{c}\text { Nitrogen } \\
\text { Fertilization }\end{array}$} & \multicolumn{2}{|c|}{$\begin{array}{l}\text { Plant height } \\
\text { (cm) }\end{array}$} & \multicolumn{2}{|c|}{$\begin{array}{c}\text { Number of } \\
\text { branches } \\
\text { /plant }\end{array}$} & \multicolumn{2}{|c|}{$\begin{array}{c}\text { Number of } \\
\text { umbels / plant }\end{array}$} & \multicolumn{2}{|c|}{$\begin{array}{c}\text { Number of } \\
\text { umbelet } \\
\text { /umbel }\end{array}$} & \multicolumn{2}{|c|}{$\begin{array}{c}\text { Number of } \\
\text { Fruits / } \\
\text { umbelet }\end{array}$} & \multicolumn{2}{|c|}{$\begin{array}{c}\text { Weight of } \\
\text { Fruits /plant } \\
\text { (gm) }\end{array}$} & \multicolumn{2}{|c|}{$\begin{array}{l}\text { Yield /plot } \\
\text { (gm) }\end{array}$} & \multicolumn{2}{|c|}{$\begin{array}{c}\text { Yield /feddan } \\
\text { (kg) }\end{array}$} & \multicolumn{2}{|c|}{$\begin{array}{l}\text { Weight of } \\
1000 \text { Fruits } \\
\text { (gm) }\end{array}$} \\
\hline & $\begin{array}{l}2006 / \\
2007\end{array}$ & $\begin{array}{l}2007 / \\
2008 \\
\end{array}$ & $\begin{array}{l}2006 / \\
2007\end{array}$ & $\begin{array}{l}2007 / \\
2008 \\
\end{array}$ & $\begin{array}{l}2006 / \\
2007 \\
\end{array}$ & $\begin{array}{l}2007 / \\
2008 \\
\end{array}$ & $\begin{array}{l}2006 / \\
2007\end{array}$ & $\begin{array}{l}2007 / \\
2008 \\
\end{array}$ & $\begin{array}{l}2006 / \\
2007\end{array}$ & $\begin{array}{l}2007 / \\
2008 \\
\end{array}$ & $\begin{array}{l}2006 / \\
2007 \\
\end{array}$ & $\begin{array}{l}2007 / \\
2008 \\
\end{array}$ & $\begin{array}{l}2006 / \\
2007\end{array}$ & $\begin{array}{l}2007 / \\
2008\end{array}$ & $\begin{array}{l}2006 / \\
2007\end{array}$ & $\begin{array}{l}2007 / \\
2008 \\
\end{array}$ & $\begin{array}{l}2006 / \\
2007\end{array}$ & $\begin{array}{l}2007 / \\
2008 \\
\end{array}$ \\
\hline 0 kg N/feddan & 104.8 & 82.4 & 10.33 & 6.33 & 49.15 & 27.30 & 15.17 & 14.60 & 7.53 & 7.40 & 16.00 & 3.48 & 201.44 & 98.564 & 211.51 & 103.49 & 4.000 & 3.579 \\
\hline $10 \mathrm{~kg} \mathrm{~N} /$ feddan & 110.2 & 92.4 & 10.88 & 8.44 & 62.28 & 35.63 & 15.51 & 13.52 & 7.66 & 7.20 & 22.81 & 6.39 & 237.77 & 132.91 & 249.66 & 139.56 & 4.461 & 3.856 \\
\hline $20 \mathrm{~kg} \mathrm{~N} /$ feddan & 114.2 & 96.4 & 12.00 & 9.77 & 69.17 & 46.60 & 15.51 & 14.13 & 8.73 & 7.06 & 25.98 & 7.70 & 306.22 & 168.91 & 321.53 & 177.36 & 4.555 & 4.104 \\
\hline $30 \mathrm{~kg}$ N/feddan & 118.7 & 98.7 & 13.33 & 11.00 & 74.53 & 49.63 & 15.77 & 14.01 & 7.46 & 7.60 & 27.15 & 9.91 & 351.00 & 204.44 & 368.55 & 214.66 & 4.765 & 4.311 \\
\hline $40 \mathrm{~kg} \mathrm{~N} /$ feddan & 122.1 & 104.2 & 14.88 & 11.88 & 88.13 & 56.68 & 15.68 & 13.85 & 8.20 & 7.60 & 33.48 & 11.42 & 441.44 & 233.88 & 463.51 & 245.58 & 4.839 & 4.420 \\
\hline $45 \mathrm{~kg} \mathrm{~N} /$ feddan & 109.6 & 90.7 & 11.11 & 8.77 & 68.66 & 47.24 & 15.22 & 14.86 & 8.53 & 8.80 & 25.98 & 9.79 & 271.00 & 139.51 & 284.55 & 146.49 & 4.050 & 3.784 \\
\hline F-test & \multicolumn{2}{|c|}{ 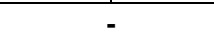 } & \multicolumn{2}{|c|}{$*$} & \multicolumn{2}{|c|}{-} & \multicolumn{2}{|c|}{ - } & \multicolumn{2}{|c|}{-} & \multicolumn{2}{|c|}{$*$} & \multicolumn{2}{|c|}{$*$} & \multicolumn{2}{|c|}{$*$} & \multicolumn{2}{|c|}{ - } \\
\hline LSD at 0.05 & \multicolumn{2}{|c|}{ NS } & \multicolumn{2}{|c|}{0.488} & \multicolumn{2}{|c|}{ NS } & \multicolumn{2}{|c|}{ NS } & \multicolumn{2}{|c|}{ NS } & \multicolumn{2}{|c|}{3.98} & \multicolumn{2}{|c|}{33.89} & \multicolumn{2}{|c|}{34.79} & \multicolumn{2}{|c|}{ NS } \\
\hline
\end{tabular}

Table (4): Effect of the interaction between plant distance and seasonal variation on growth and productivity of caraway (Carum carvi $\mathrm{L}$.)

\begin{tabular}{|c|c|c|c|c|c|c|c|c|c|c|c|c|c|c|c|c|c|c|}
\hline \multirow[b]{2}{*}{ Plant distance } & \multicolumn{2}{|c|}{$\begin{array}{l}\text { Plant height } \\
\text { (cm) }\end{array}$} & \multicolumn{2}{|c|}{$\begin{array}{c}\text { Number of } \\
\text { branches/ } \\
\text { plant }\end{array}$} & \multicolumn{2}{|c|}{$\begin{array}{c}\text { Number of } \\
\text { umbels /plant }\end{array}$} & \multicolumn{2}{|c|}{$\begin{array}{c}\text { Number of } \\
\text { umbelet/ } \\
\text { umbel }\end{array}$} & \multicolumn{2}{|c|}{$\begin{array}{l}\text { Number of } \\
\text { fruits / } \\
\text { umbelet }\end{array}$} & \multicolumn{2}{|c|}{$\begin{array}{c}\text { Weight of } \\
\text { fruits /plant } \\
\text { (gm) }\end{array}$} & \multicolumn{2}{|c|}{ Yield /plot (gm) } & \multicolumn{2}{|c|}{$\begin{array}{c}\text { Yield /feddan } \\
\text { (kg) }\end{array}$} & \multicolumn{2}{|c|}{$\begin{array}{l}\text { Weight of } \\
1000 \text { fruits } \\
\text { (gm) }\end{array}$} \\
\hline & $\begin{array}{l}2006 / \\
2007\end{array}$ & $\begin{array}{l}2007 / \\
2008\end{array}$ & $\begin{array}{l}2006 / \\
2007\end{array}$ & $\begin{array}{l}2007 / \\
2008\end{array}$ & $\begin{array}{l}2006 / \\
2007\end{array}$ & $\begin{array}{l}2007 / \\
2008\end{array}$ & $\begin{array}{l}2006 / \\
2007\end{array}$ & $\begin{array}{l}2007 / \\
2008\end{array}$ & $\begin{array}{l}2006 / \\
2007\end{array}$ & $\begin{array}{l}2007 / \\
2008\end{array}$ & $\begin{array}{l}2006 / \\
2007\end{array}$ & $\begin{array}{l}2007 / \\
2008\end{array}$ & $\begin{array}{l}2006 / \\
2007\end{array}$ & $\begin{array}{l}2007 / \\
2008\end{array}$ & $\begin{array}{l}2006 / \\
2007\end{array}$ & $\begin{array}{l}2007 / \\
2008\end{array}$ & $\begin{array}{l}2006 / \\
2007\end{array}$ & $\begin{array}{l}2007 / \\
2008\end{array}$ \\
\hline $20 \mathrm{~cm}$ & 121.6 & 99.2 & 11.00 & 8.00 & 48.95 & 28.36 & 15.02 & 13.90 & 8.33 & 7.73 & 17.89 & 6.48 & 248.44 & 123.22 & 260.86 & 129.38 & 3.938 & 3.491 \\
\hline $40 \mathrm{~cm}$ & 113.7 & 94.6 & 12.05 & 9.44 & 71.05 & 47.76 & 15.53 & 14.91 & 7.86 & 8.10 & 27.76 & 8.25 & 300.72 & 172.11 & 315.75 & 180.71 & 4.521 & 4.091 \\
\hline $60 \mathrm{~cm}$ & 104.5 & 88.6 & 13.22 & 10.66 & 85.96 & 55.42 & 15.88 & 13.67 & 7.86 & 7.00 & 30.05 & 9.62 & 355.27 & 193.78 & 373.04 & 203.47 & 4.876 & 4.446 \\
\hline F-test & \multicolumn{2}{|c|}{-} & \multicolumn{2}{|c|}{. } & \multicolumn{2}{|c|}{. } & & \multicolumn{2}{|c|}{ 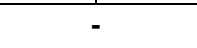 } & \multicolumn{2}{|c|}{ 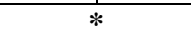 } & \multicolumn{2}{|c|}{$*$} & \multicolumn{2}{|c|}{$*$} & \multicolumn{2}{|c|}{-} \\
\hline LSD at 0.05 & \multicolumn{2}{|c|}{ NS } & \multicolumn{2}{|c|}{ NS } & \multicolumn{2}{|c|}{ NS } & \multicolumn{2}{|c|}{ NS } & \multicolumn{2}{|c|}{ NS } & \multicolumn{2}{|c|}{3.16} & \multicolumn{2}{|c|}{23.97} & \multicolumn{2}{|c|}{27.66} & \multicolumn{2}{|c|}{ NS } \\
\hline
\end{tabular}


Table (5): Effect of the interaction between plant distance and nitrogen fertilization on growth and productivity of caraway (Carum carvi L.)

\begin{tabular}{|c|c|c|c|c|c|c|c|c|c|c|}
\hline & \multirow{2}{*}{$\begin{array}{c}\text { Plant } \\
\text { height }(\mathrm{cm})\end{array}$} & \multirow{2}{*}{$\begin{array}{c}\text { Number of } \\
\text { branches / } \\
\text { plant }\end{array}$} & \multirow{2}{*}{$\begin{array}{c}\text { Number of } \\
\text { umbels / } \\
\text { plant }\end{array}$} & \multirow{2}{*}{$\begin{array}{c}\text { Number of } \\
\text { umbelet / } \\
\text { umbel }\end{array}$} & \multirow{2}{*}{$\begin{array}{l}\text { Number of } \\
\text { Fruits / } \\
\text { umbelet }\end{array}$} & \multirow{2}{*}{$\begin{array}{l}\text { Weight of } \\
\text { Fruits /plant } \\
\text { (gm) }\end{array}$} & \multirow{2}{*}{$\begin{array}{c}\text { Yield / } \\
\text { plot (gm) }\end{array}$} & \multirow{2}{*}{$\begin{array}{c}\text { Yield / } \\
\text { feddan (kg) }\end{array}$} & \multirow{2}{*}{$\begin{array}{l}\text { Weight of } \\
1000 \text { Fruits } \\
\text { (gm) }\end{array}$} \\
\hline $\begin{array}{c}\text { Plant } \\
\text { distance }\end{array}$ & $\begin{array}{c}\text { Nitrogen } \\
\text { Fertilization }\end{array}$ & & & & & & & & & \\
\hline $20 \mathrm{~cm}$ & $\begin{array}{c}0 \mathrm{~kg} \mathrm{~N} / \text { feddan } \\
10 \mathrm{~kg} \mathrm{~N} / \text { feddan } \\
20 \mathrm{~kg} \mathrm{~N} / \text { feddan } \\
30 \mathrm{~kg} \mathrm{~N} / \text { feddan } \\
40 \mathrm{~kg} \mathrm{~N} / \text { feddan } \\
45 \mathrm{~kg} \mathrm{~N} / \text { feddan }\end{array}$ & \begin{tabular}{|l|l|}
102.16 \\
108.16 \\
112.66 \\
116.50 \\
120.00 \\
103.16
\end{tabular} & \begin{tabular}{|c|}
7.00 \\
8.33 \\
9.833 \\
11.00 \\
11.833 \\
9.00
\end{tabular} & \begin{tabular}{|l|}
26.800 \\
32.133 \\
38.300 \\
39.267 \\
51.167 \\
44.300
\end{tabular} & \begin{tabular}{|l|l|}
14.467 \\
14.583 \\
14.667 \\
14.133 \\
14.050 \\
14.867
\end{tabular} & $\begin{array}{l}7.90 \\
7.10 \\
8.20 \\
6.90 \\
8.50 \\
9.60\end{array}$ & \begin{tabular}{|c|}
7.280 \\
8.813 \\
10.803 \\
12.317 \\
17.212 \\
16.709
\end{tabular} & \begin{tabular}{l|}
135.833 \\
151.517 \\
204.833 \\
212.167 \\
260.167 \\
150.500
\end{tabular} & \begin{tabular}{l|}
142.62 \\
159.09 \\
215.07 \\
222.77 \\
273.17 \\
158.02
\end{tabular} & \begin{tabular}{l|}
2.994 \\
3.631 \\
3.854 \\
3.969 \\
4.243 \\
3.595
\end{tabular} \\
\hline $40 \mathrm{~cm}$ & $\begin{array}{c}0 \mathrm{~kg} \text { N/feddan } \\
10 \mathrm{~kg} \text { N/feddan } \\
20 \mathrm{~kg} \mathrm{~N} / \text { feddan } \\
30 \mathrm{~kg} \mathrm{~N} / \text { feddan } \\
40 \mathrm{~kg} \text { N/feddan } \\
45 \mathrm{~kg} \text { N/feddan }\end{array}$ & \begin{tabular}{|c|}
97.83 \\
102.83 \\
104.50 \\
108.00 \\
112.33 \\
99.83 \\
\end{tabular} & \begin{tabular}{|c|}
8.333 \\
9.833 \\
10.667 \\
12.00 \\
13.667 \\
10.00 \\
\end{tabular} & \begin{tabular}{|l|}
39.500 \\
46.767 \\
63.633 \\
65.617 \\
79.200 \\
61.733 \\
\end{tabular} & \begin{tabular}{|l|l|}
15.667 \\
14.900 \\
14.700 \\
16.183 \\
15.100 \\
14.800 \\
\end{tabular} & $\begin{array}{l}8.00 \\
8.20 \\
7.40 \\
7.30 \\
8.20 \\
8.80\end{array}$ & \begin{tabular}{|l|l|}
10.235 \\
16.013 \\
18.974 \\
20.541 \\
24.144 \\
18.160 \\
\end{tabular} & \begin{tabular}{|l|}
140.167 \\
171.803 \\
229.038 \\
302.667 \\
364.997 \\
209.833 \\
\end{tabular} & \begin{tabular}{|l|l|}
147.17 \\
180.39 \\
240.49 \\
317.80 \\
383.24 \\
220.32 \\
\end{tabular} & \begin{tabular}{l|}
3.988 \\
4.335 \\
4.445 \\
4.697 \\
4.587 \\
3.782 \\
\end{tabular} \\
\hline $60 \mathrm{~cm}$ & $\begin{array}{l}0 \mathrm{~kg} \text { N/feddan } \\
10 \mathrm{~kg} \text { N/feddan } \\
20 \mathrm{~kg} \mathrm{~N} / \text { feddan } \\
30 \mathrm{~kg} \mathrm{~N} / \text { feddan } \\
40 \mathrm{~kg} \text { N/feddan } \\
45 \mathrm{~kg} \text { N/feddan }\end{array}$ & \begin{tabular}{|c|}
81.00 \\
93.00 \\
98.83 \\
101.83 \\
107.16 \\
97.667
\end{tabular} & \begin{tabular}{|c|}
9.667 \\
10.833 \\
12.167 \\
13.50 \\
14.667 \\
10.833
\end{tabular} & \begin{tabular}{|l|}
48.383 \\
67.983 \\
71.733 \\
81.367 \\
86.867 \\
67.833
\end{tabular} & \begin{tabular}{|l|}
14.533 \\
14.067 \\
15.100 \\
14.367 \\
15.167 \\
15.467
\end{tabular} & $\begin{array}{l}6.50 \\
7.00 \\
8.10 \\
8.40 \\
7.00 \\
7.60\end{array}$ & $\begin{array}{l}11.721 \\
18.985 \\
20.756 \\
22.754 \\
26.017 \\
18.786\end{array}$ & \begin{tabular}{|l|}
174.013 \\
232.723 \\
278.833 \\
318.330 \\
387.833 \\
255.445
\end{tabular} & \begin{tabular}{l|}
182.71 \\
244.36 \\
292.77 \\
334.25 \\
407.22 \\
268.21
\end{tabular} & $\begin{array}{l}4.386 \\
4.509 \\
4.690 \\
4.947 \\
5.058 \\
4.375\end{array}$ \\
\hline \multicolumn{2}{|r|}{ F-test } & - & - & - & - & - & $*$ & $*$ & $*$ & - \\
\hline \multicolumn{2}{|c|}{ LSD at 0.05} & NS & NS & NS & NS & NS & 4.70 & 41.52 & 41.05 & NS \\
\hline
\end{tabular}

Table (6): Effect of plant distance and nitrogen fertilization on oil (\%) in crushed fruits of caraway.

\begin{tabular}{|c|c|c|c|c|c|}
\hline \multicolumn{2}{|l|}{ Fertilizer } & $20 \mathrm{~cm}$ & $40 \mathrm{~cm}$ & $60 \mathrm{~cm}$ & Mean \\
\hline $\begin{array}{l}0 \mathrm{~kg} \text { nitrogen } \\
10 \mathrm{~kg} \text { nitroge } \\
20 \mathrm{~kg} \text { nitroge } \\
30 \mathrm{~kg} \text { nitroge } \\
40 \mathrm{~kg} \text { nitroge } \\
45 \mathrm{~kg} \text { nitroge }\end{array}$ & $\begin{array}{l}\text { ddan } \\
\text { feddan } \\
\text { feddan } \\
\text { feddan } \\
\text { feddan } \\
\text { feddan } \\
\end{array}$ & \begin{tabular}{|l|}
$\mathbf{1 . 8 3 3}$ \\
$\mathbf{2 . 8 6 7}$ \\
$\mathbf{3 . 2 0 0}$ \\
$\mathbf{3 . 2 0 0}$ \\
$\mathbf{3 . 3 3 3}$ \\
$\mathbf{2 . 7 3 3}$ \\
\end{tabular} & \begin{tabular}{l|}
2.573 \\
3.333 \\
3.500 \\
4.400 \\
4.067 \\
$\mathbf{3 . 5 3 3}$ \\
\end{tabular} & $\begin{array}{l}\mathbf{2 . 7 3 3} \\
\mathbf{3 . 4 6 7} \\
\mathbf{3 . 5 3 3} \\
\mathbf{4 . 2 0 0} \\
\mathbf{4 . 4 3 3} \\
\mathbf{3 . 2 6 7} \\
\end{array}$ & $\begin{array}{l}\mathbf{2 . 3 8 0} \\
\mathbf{3 . 2 2 2} \\
\mathbf{3 . 4 1 1} \\
\mathbf{3 . 9 3 3} \\
\mathbf{3 . 9 4 4} \\
\mathbf{3 . 1 7 8} \\
\end{array}$ \\
\hline \multicolumn{2}{|c|}{ Mean } & 2.861 & 3.568 & 3.606 & \\
\hline \multirow{3}{*}{ LSD at 0.05} & Plant Distance & \multicolumn{4}{|c|}{0.349} \\
\hline & Nitrogen Fertilizer & \multicolumn{4}{|c|}{0.712} \\
\hline & Distance X Fertilizer & \multicolumn{4}{|c|}{ NS } \\
\hline
\end{tabular}




\subsection{The interaction between season and nitrogen fertilization}

Data presented in Table (3) reveal that plant height, weight of seeds per plant (gm), fruit yield per plot (gm) and fruit yield per feddan (kg) were significantly affected by the interaction between seasons and nitrogen fertilization. These results may be due to the environmental conditions. with $40 \mathrm{~kg}$ nitrogen/plant). In the case of weight of fruits per plant $(\mathrm{gm})$ no significant differences were obtained when the distance was $40 \mathrm{~cm}$ distance between plants and plants received $40 \mathrm{~kg}$ nitrogen/plant) or at $60 \mathrm{~cm}$ distance between plants and plants received $30 \mathrm{~kg}$ nitrogen/plant, and also at $60 \mathrm{~cm}$ distance between plants and

Table (7): Effect of plant distance and nitrogen fertilization on oil yield/feddan $(L)$ in crushed fruits of caraway .

\begin{tabular}{|c|c|c|c|c|c|}
\hline \multicolumn{2}{|c|}{$\begin{array}{l}\text { Distance } \\
\text { Fertilizer }\end{array}$} & $20 \mathrm{~cm}$ & $40 \mathrm{~cm}$ & $60 \mathrm{~cm}$ & Mean \\
\hline $\begin{array}{l}0 \mathrm{~kg} \text { nitroger } \\
10 \mathrm{~kg} \text { nitroge } \\
20 \mathrm{~kg} \text { nitroge } \\
30 \mathrm{~kg} \text { nitroge } \\
40 \mathrm{~kg} \text { nitroge } \\
45 \mathrm{~kg} \text { nitroge }\end{array}$ & $\begin{array}{l}\text { eddan } \\
\text { feddan } \\
\text { feddan } \\
\text { feddan } \\
\text { feddan } \\
\text { feddan }\end{array}$ & $\begin{array}{l}1.677 \\
3.579 \\
4.340 \\
4.550 \\
6.426 \\
2.108\end{array}$ & \begin{tabular}{|c|}
2.521 \\
4.203 \\
6.741 \\
10.472 \\
11.136 \\
5.960
\end{tabular} & \begin{tabular}{r|}
3.357 \\
5.838 \\
7.287 \\
10.920 \\
12.145 \\
6.486
\end{tabular} & $\begin{array}{l}2.518 \\
4.540 \\
6.123 \\
8.647 \\
9.902 \\
4.852\end{array}$ \\
\hline \multicolumn{2}{|r|}{ Mean } & 3.780 & 6.839 & 7.672 & \\
\hline \multirow{3}{*}{ LSD at 0.05} & Distance & \multicolumn{4}{|c|}{2.047} \\
\hline & Fertilizer & \multicolumn{4}{|c|}{2.606} \\
\hline & Distance X Fertilizer & \multicolumn{4}{|c|}{ NS } \\
\hline
\end{tabular}

\subsection{The interaction between plant distance and nitrogen fertilization}

Data presented in Tables (5, 6 and 7) reveal that weight of seeds per plant (gm), fruit yield per plot (gm) and fruit yield per feddan (kg) were significantly affected by the interaction between plant distance and nitrogen fertilization. The maximum values were obtained from planting at $60 \mathrm{~cm}$ between the plants and applying $40 \mathrm{~kg}$ nitrogen per feddan. No significant differences were obtained between the planting distance of 40 $\mathrm{cm}$ with fertilization at $40 \mathrm{~kg}$ nitrogen/plant and the distance of $60 \mathrm{~cm}$ distance between plants plants received $40 \mathrm{~kg}$ nitrogen/plant).

Fig. (1) shows a curve estimating the interaction between plant distance and nitrogen fertilization for yield/feddan of caraway. The response rate of the first stage was positive over all treatments. The increments per one fertilizer $\mathrm{kg}$ were $6.763,10.28$ and $10.00 \mathrm{~kg}$ with planting distances of 20, 40 and $60 \mathrm{~cm}$, respectively. The response rate of second stage was negative over all the treatments. The reduction per one fertilizer $\mathrm{kg}$ was equal $0.114,0.147$ and 0.148 kgin 20,40 and $60 \mathrm{~cm}$, respectively.

We can determine the best level of nitrogen ( 40 $\mathrm{kg}$ nitrogen/feddan) that gives the maximum yield

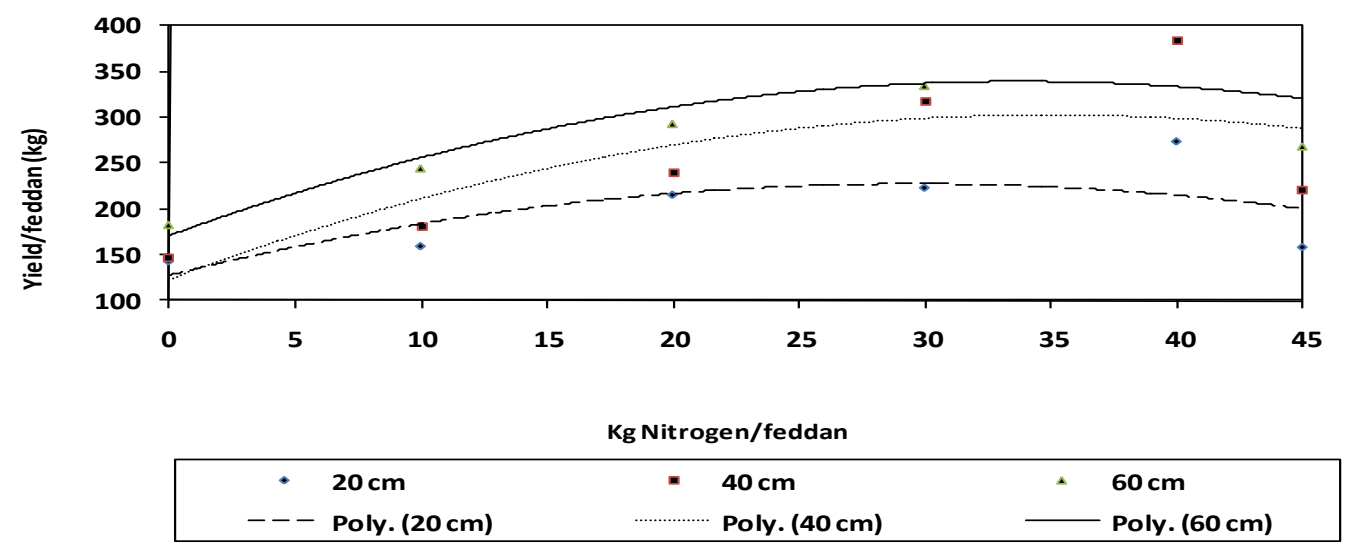


value from the point of maximum curvature, and we can predict from the graph directly the yield values at any level of nitrogen fertilization within the range.

$$
\begin{aligned}
& \text { The predicted equations: } \\
& \mathrm{Y}(20 \mathrm{~cm})=127.4+6.763 \mathrm{x}-0.114 \mathrm{x}^{2} \\
& \mathrm{Y}(40 \mathrm{~cm})=122.8+10.28 \mathrm{x}-0.147 \mathrm{x}^{2} \\
& \mathrm{Y}(60 \mathrm{~cm})=170.6+10.00 \mathrm{x}-0.148 \mathrm{x}^{2}
\end{aligned}
$$

3.7. The interaction between season, plant distance and nitrogen fertilization

Data presented in Table (2) reveal that plant height was significantly affected by the interaction between plant distance, nitrogen fertilization and seasons. The maximum values were obtained by using a planting distance of $20 \mathrm{~cm}$ and fertilization with $40 \mathrm{~kg}$ nitrogen/feddan. No significant differences were obtained when using $(20 \mathrm{~cm}$ between plants and $30 \mathrm{~kg}$ nitrogen/plant) and (20 $\mathrm{cm}$ between plants and $40 \mathrm{~kg}$ nitrogen/plant). These results may be due to the nature of plant growth and environmental conditions.

According to the above mentioned results, it could be stated that a plant spacing of $40 \mathrm{~cm}$ and $40 \mathrm{~kg}$ nitrogen/feddan produced the maximum yield.

\section{REFERENCES}

Ahmed E. T. (1997). Influence of plant distance and some phosphorus fertilization sources on black cumin (Nigella sativa L.) plants. Assiut J. of Agric. Sci., 28(2): 39-56.

Badran F. S. and Hafez M. H. (2002). Influence of planting date and plant density on Nigella sativa L. plants. Proceedings of the Second Conference of Sustainable Agricultural Development. Fayoum Faculty of Agriculture, 8-10 May.

Barreyro R. A., Sanchez-Vallduvi G. E., Chamorro A. M., Bezus R. and Petruccelli V. G. (1993). Increase in coriander (Coriandrum sativum L.) biomass and essential oil content as a result of nitrogen fertilizer application. Revista de la Facultad de Agronomia la plata, 69(1): 59-61. (CAB. Abst).

Bhati D. S. and Shaktawat M. S. (1994). Effect of sowing date, row spacing and nitrogen on quality parameters of coriander (Coriandrum sativum L.). Progressive Hort., 26 (1/2):14-18.

British Pharmacopoeia (1963). Determination of Volatile Oil in Drugs. The Pharmaceutical Press, London, U.K.

El-Mansi A., Mostafa A. Baker M. and Sonbol E.R. (1970). Effect of fertilization and irrigation on yield, oil content of coriander and its physiochemical properties. Res. Bull. No. 168, Ain Shams Univ., Egypt.

Hussien M. S. (1995). Response of growth, yield and essential oil of coriander and dill to different nitrogen sources. Egypt. J. Hort., 22(1): 1-10.

Radwan A. A. (1980). Effect of Some Cultural Treatments on the Growth of Some Umbelliferous plants and their active Constituents. M. Sc. Thesis, Fac. Agric., Ain Shams Univ., Egypt.

Rahman M. O., Babu R. S. and Rao B. R. B. (1990). Influence of different levels of nitrogen on coriander (Coriandrum sativum L.) on Alfisol. Journal of Research APAU, 18(4):346-348.

Snedecor G. W. and Cochran W. G. (1980). Statistical Methods. $7^{\text {th }}$ edition, Iowa State University Press, Ames, Iowa.

Tiwari R. J. and Banafar R. N. S. (1995). Application of nitrogen and phosphorus increases seed yield and essential oil of coriander. Indian Cocoa, Arecanut and Spices Journal, 19(2): 51-55 (C.F. Hort. Abst., 66:7988)

$$
\begin{aligned}
& \text { إستجابة محصول نبات الكراوية لمعاملات مسافات الزراعة والتسميد النيتروجينى } \\
& \text { عبد الغفور عوض السيد - سعيد محم نصر*- منى أحمد درويش - مروه محم سليمان } \\
& \text { قسم بساتين الزينة - كلية زراعة - جامعة القاهرة - الجيزة - مصر }
\end{aligned}
$$

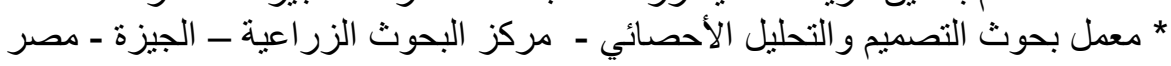

$$
\begin{aligned}
& \text { ملخص الخص مانر } \\
& \text { اقيمت تجربتان حقليتان فى مزر عه كلية الزراعة- جامعة القاهرة خلال موسمي الزراعة }
\end{aligned}
$$

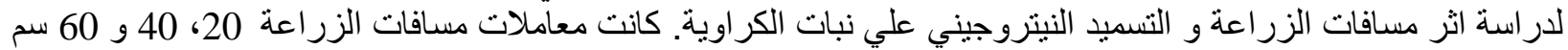

$$
\begin{aligned}
& \text { بين النباتات. أمامعاملات التسميد النيتروجينى فكانت 0، 10، 20، 30، } \\
& \text { أظهرت النتائج ان اعلى طول للنباتات تم الحصول علي عند استخدام المسافة } 20 \text { سم بين النباتات بينما حققت صفات صفات عدد } \\
& \text { الفروع الرئيسية للنبات، عدد النورات على النبات، وزن الثمار للنبات بالجرام، محصول الثمار للقطعة التجريبية بالجر ام، }
\end{aligned}
$$




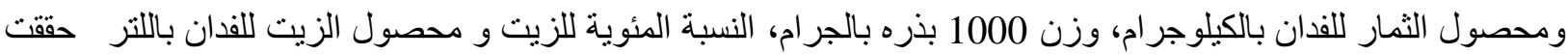

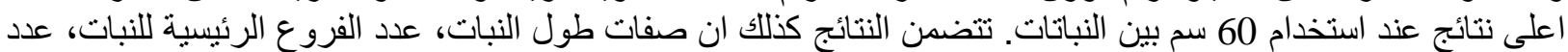

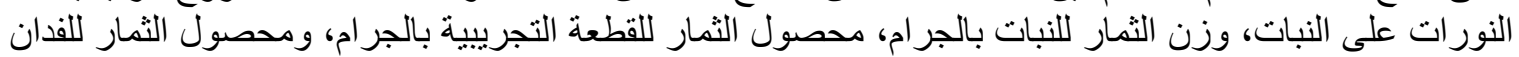

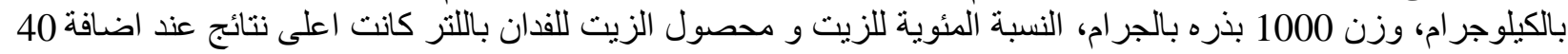

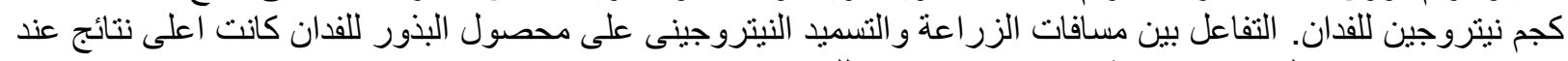

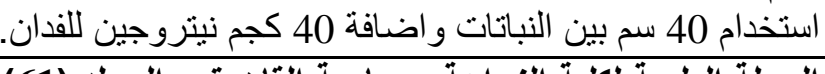

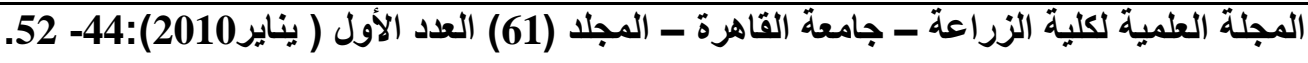

